\title{
Differential Diagnosis of Malignant Biliary Tract Cancer from Benign Tissues using Apparent Diffusion Coefficient Measurements with Diffusion Weighted Imaging in Asians
}

\author{
Xu-Ya Zhao*, Shi Zhou, Da-Zhi Wang, Wei He, Jun-Xiang Li, Shuai Zhang
}

\begin{abstract}
Background: The aim of this meta-analysis was to assess the efficacy of the apparent diffusion coefficient (ADC) value of diffusion-weighted MRI (DWI) for differentiating biliary tract cancer (BTC) from benign biliary tract diseases in Asians. Materials and Methods: We systematically searched Embase and PubMed prior to December 2014. Eight studies conducted in Asians met our predetermined inclusion criteria. Results: Our meta-analysis results showed that ADC values in BTC tissues were significantly lower than in benign biliary tract tissues (SMD $=-1.54,95 \%$ CI: $-1.75 \sim 1.33, P<0.001)$. Subgroup analysis based on the MRI machine type showed that the ADC values were consistent, accurate and reliable in the diagnosis of BTC when comparing cancer tissue vs. benign tissue under the Siemens 1.5 T/3.0 T, Philips 1.5 T/3.0 T, GE 1.5 T, and Toshiba 1.5 T types, respectively (all $P<0.05)$. Further, ADC values were still consistent and accurate in the differential diagnosis of BTC under the b value of 800 and $1000 \mathrm{~s} / \mathrm{mm}^{2}$ (all $P<0.05$ ). Conclusions: Our findings supported potential clinical applications of DWI ADC values in differentiating BTC from benign biliary tract diseases in Asians.
\end{abstract}

Keywords: Biliary tract cancer - diffusion-weighted magnetic resonance imaging - meta-analysis

Asian Pac J Cancer Prev, 16 (14), 6135-6140

\section{Introduction}

Biliary tract cancer (BTC) remains an intractable and lethal malignancy at presentation, encompassing cancers of the gallbladder, extrahepatic bile duct and ampulla of Vater (Furuse et al., 2012; Morizane et al., 2013). Being estimated, BTC ranks the sixth most common alimentary tract cancer in the United States, and gallbladder cancer is the most common biliary tract tumor worldwide (Gourgiotis et al., 2008; Castro et al., 2013). Characterized by a wide geographic variation, BTC is relatively infrequent in Europe and North America, but increasing trends in BTC mortality rates were observed in some areas of Asia; and the decreased mortality rate of BTC were more favorable in middle-aged women (Randi et al., 2009). BTC has been reported to be easily spread to the lymph nodes and surrounding normal structures and the prognostic outcome of BTC is extremely poor with the 5-year survival rate less than $5 \%$ in most cases (Charatcharoenwitthaya et al., 2008). More previous analyses have also noted, substantial geographic, ethnic variations and gender are involved in each of the specific cancer sites (Hsing et al., 2008; Shin et al., 2010). Early and accurate diagnostic methods in BTC are therefore important for the prevention of increased morbidity in Asia especially as well as delayed or ineffective treatment (Lee et al., 2014a). Changes in diagnosis and confirmation of the disease, followed by increased availability of sophisticated diagnostic techniques may be partly responsible for the alternation in BTC mortality (Vilana et al., 2010; Wen et al., 2010). Magnetic resonance imaging (MRI) has been used in BTC, and diffusion-weighted MRI (DWI) has also been introduced (Malayeri et al., 2011; Tan and Lim, 2013).

DWI, a functional MRI technique sensitive to water molecular diffusion, that is the Brownian motion of water protons in biologic tissues, can provide us with a deeper insight into micro-structural tissue changes (Wu et al., 2013; Lambrecht et al., 2014). DWI could characterize specific tissue properties without any harm to patients, especially benefit for those who are at risk for complications of a biopsy procedure (Heijmen et al., 2012). More importantly, with its lack of ionizing radiation but high contrast and spatial resolution, DWI has become a promising technique for diagnosis of benign and malignant tumors of various organs (Akay et al., 2013; Li et al., 2014). By providing qualitative and quantitative information about tissue cellularity, DWI has been increasingly used in oncologic imaging for monitoring response to treatment (Kyriazi et al., 2010). The different diffusion ability of extracellular and intracellular water molecule, with more freedom for extracellular water molecules, can be detected by DWI, therefore reflective of tissue organizational features; 


\section{Xu-Ya Zhao et al}

especially cellularity can be acquired (Khoo et al., 2011). As a major quantitative parameter of DWI, the apparent diffusion coefficient (ADC) value can reflect the thermal diffusion of water molecules in biological tissues (Onur et al., 2012). Water motion degree is proportional to DWI signal attenuation degree, thus more cellular solid tumors with hyper-cellularity show relatively higher signal intensities and lower ADC values (Lee et al., 2012). DWI which is first used in brain disease detection has been widely used for evaluation of the solid abdominal and pelvic organs, including the liver, kidneys, pancreas, prostate, uterus and ovaries (Low et al., 2009). Possible reason for its widespread could be explained by its ready compatibility with most existing MRI protocols and needless of extra scanner hardware, prolonged scanning times or intravenous contrast material administration (Attariwala and Picker, 2013).

Accordingly, we postulated that DWI may be valuable in differentiating malignancies from benign biliary tract diseases among Asian populations, for the present situation of increasing trends in BTC mortality in Asians. Several studies have illustrated that malignant BTC may be differentiated from benign lesions via the ADC quantified by DWI (Kang et al., 2013; Kim et al., 2013a; Lee et al., 2014a). Nevertheless, there are also studies revealed that the characterization of biliary tract masses as benign or malignant could not be achieved only based on DWI, thus its diagnostic capabilities in BTC still remains highly controversial (Kim et al., 2012; Baheti et al., 2014). So we performed this meta-analysis to assess the feasibility of DWI in association with ADC measurement as diagnostic tool in differential diagnosis of malignancies from benign biliary tract diseases among Asian populations.

\section{Materials and Methods}

\section{Search strategy}

We systematically searched Embase and PubMed prior to December 2014, focusing on the diagnostic potential of DWI ADC values in differential diagnosis of BTC from benign biliary tract diseases. The search strategy used combinations of Mesh terms for "Diffusion Magnetic Resonance Imaging" and "Biliary Tract Neoplasms" with synonyms for ("Diffusion MRI" OR "Diffusion Weighted MRI” OR “Diffusion Magnetic Resonance Imaging” OR "diffusion-weighted magnetic resonance imaging" OR "MRI-DWI" OR "diffusion-weighted imaging" OR “diffusion-weighted-MRI") with ("Biliary Tract" OR "cholangio" OR "gallbladder" OR "bile duct" OR "cholecystic") and ("cancer" OR "tumor" OR "tumour" OR “carcinoma” OR “neoplasm" OR "malignant”) occurred in the title or abstract. No restriction on language was preset. We also manually screened published bibliographies.

\section{Selection criteria}

After titles and abstracts screening for all relevant studies, we selected potential articles and reviewing full text to judge whether they were really eligible. The selection of studies for meta-analysis was performed by two independent investigators. The inclusion criteria were: (1) evaluation of the relationship of ADC values in DWI with the discrimination of BTC; (2) original diagnostic studies or observational studies conducted in an Asian populations; (3) control subjects with benign biliary tract lesions; (4) all BTC patients were confirmed by laboratory examination, ultrasound or computed tomography with pathological confirmation (Chen et al., 2008); (5)

Table 1. Main Characteristics and Methodological Quality of All Eligible Studies

\begin{tabular}{|c|c|c|c|c|c|c|c|c|c|c|c|}
\hline \multirow[b]{2}{*}{ Authors } & \multirow{2}{*}{ Year } & \multirow{2}{*}{$\frac{\text { Inclusion }}{\text { period }}$} & \multirow[t]{2}{*}{ Country } & \multicolumn{2}{|c|}{ Samples } & \multicolumn{2}{|c|}{ Age (years) } & \multicolumn{2}{|c|}{$\operatorname{Sex}(M / F)$} & \multirow{2}{*}{$\begin{array}{c}\text { MRI } \\
\text { Machine } \\
\text { type }\end{array}$} & \multirow{2}{*}{$\begin{array}{c}\text { Maximum } \\
\text { b value } \\
\left(\mathrm{s} / \mathrm{mm}^{2}\right)\end{array}$} \\
\hline & & & & Case & Control & Case & Control & Case & Control & & \\
\hline Lee & 2014 & $\begin{array}{l}2009- \\
2010\end{array}$ & Korea & 36 & 90 & \multicolumn{2}{|c|}{$59.8(19 \sim 80)$} & \multicolumn{2}{|c|}{$79 / 47$} & $\begin{array}{c}\text { Siemens } \\
1.5 \mathrm{~T}\end{array}$ & 800 \\
\hline & & & & & & & & & & $\begin{array}{c}\text { Siemens } \\
3.0 \mathrm{~T}\end{array}$ & 800 \\
\hline Kim & 2013 & $\begin{array}{c}2007- \\
2010\end{array}$ & Korea & 39 & 36 & $\begin{array}{c}62.5(41 \\
\sim 81) \\
\end{array}$ & $\begin{array}{c}61(38 \sim \\
83) \\
\end{array}$ & $19 / 20$ & $22 / 14$ & GE $1.5 \mathrm{~T}$ & 1000 \\
\hline Kang & 2013 & $\begin{array}{l}2008- \\
2011\end{array}$ & Korea & 19 & 14 & $\begin{array}{c}66(38 \sim \\
84)\end{array}$ & $\begin{array}{c}60.7(39 \sim \\
80)\end{array}$ & $10 / 9$ & $9 / 5$ & $\begin{array}{c}\text { Philips } \\
3.0 \mathrm{~T}\end{array}$ & 800 \\
\hline Solak A & 2013 & $\begin{array}{c}2011- \\
2011 \\
\end{array}$ & Turkey & 7 & 9 & \multicolumn{2}{|c|}{56.2} & $1 / 6$ & $4 / 5$ & $\begin{array}{c}\text { Siemens } \\
1.5 \mathrm{~T} \\
\end{array}$ & 800 \\
\hline \multirow[t]{2}{*}{$\begin{array}{c}\text { Yoshioka } \\
\text { M }\end{array}$} & 2013 & $\begin{array}{l}2006- \\
2011\end{array}$ & Japan & 22 & 7 & \multirow{2}{*}{\multicolumn{2}{|c|}{$64(50 \sim 82)$}} & \multirow{2}{*}{\multicolumn{2}{|c|}{$20 / 20$}} & GE $1.5 \mathrm{~T}$ & 1000 \\
\hline & & & & 22 & 11 & & & & & GE $1.5 \mathrm{~T}$ & 1000 \\
\hline Ogawa & 2012 & $\begin{array}{c}2005- \\
2010 \\
\end{array}$ & Japan & 36 & 117 & $71 \pm 10$ & $57 \pm 15$ & $18 / 18$ & $60 / 57$ & $\begin{array}{c}\text { Toshiba } \\
1.5 \mathrm{~T} \\
\end{array}$ & 1000 \\
\hline Irie & 2011 & $\begin{array}{l}2007- \\
2010 \\
\end{array}$ & Japan & 13 & 10 & \multicolumn{2}{|c|}{69 (40 85) } & \multicolumn{2}{|c|}{$6 / 17$} & $\begin{array}{c}\text { Siemens } \\
1.5 \mathrm{~T} \\
\end{array}$ & 1000 \\
\hline Sugita & 2009 & $\begin{array}{l}2005- \\
2007\end{array}$ & Japan & 15 & 14 & $\begin{array}{c}75(59 \sim \\
92)\end{array}$ & $\begin{array}{c}64(37 \sim \\
88)\end{array}$ & $9 / 6$ & $11 / 3$ & $\begin{array}{c}\text { Toshiba } \\
1.5 \mathrm{~T}\end{array}$ & 1000 \\
\hline
\end{tabular}

*M: male; F: female; MRI: magnetic resonance imaging 


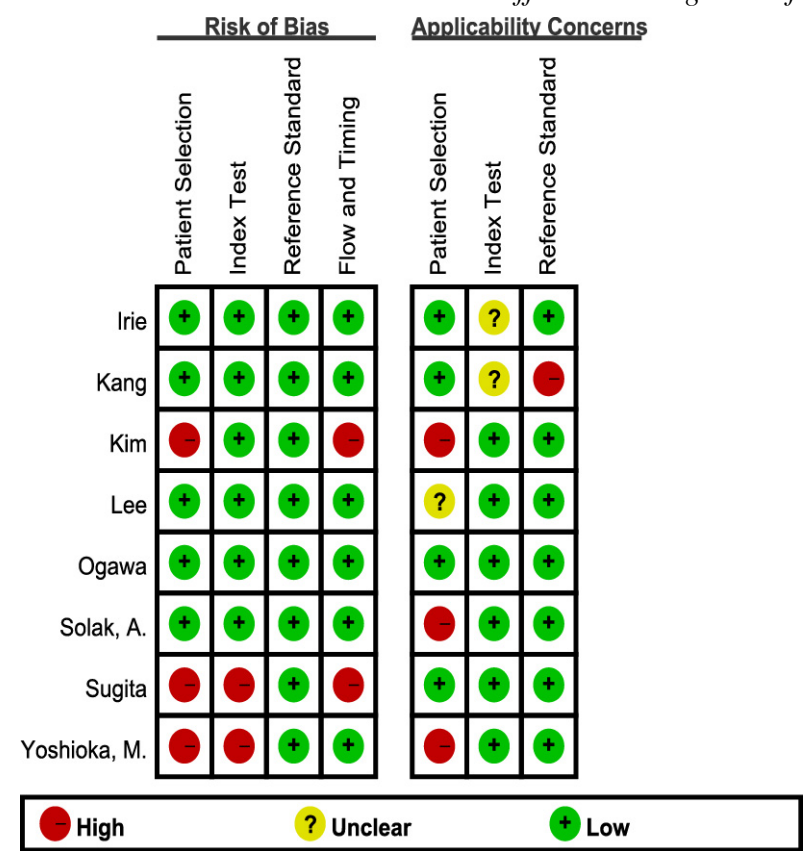

Figure 1. Risk of Bias Summary: Review Authors' Judgements About Each risk of Bias Item for Each Included Study

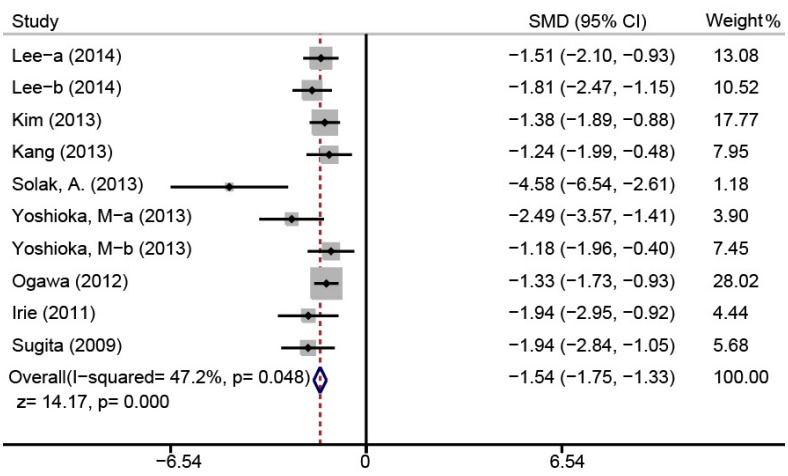

Figure 2. Forest Plots on the Difference of ADC Value between Benign and Malignant Biliary Tract Tumors

studies with the most details or the most recent publish date. Exclusion criteria were: (1) insufficient dataset information; (2) study subjects were not confirmed by a standard clinical examination; (3) studies in which crude data could not be extracted. For studies with multiple publications, the most recent publication was chosen.

\section{Data extraction and quality assessment}

Two investigators reviewed the included studies in duplicate and extract all relevant information such as: first author, publication year, country and ethnicity, samples, ages and genders, MRI machine type, b value, malignant tumors and benign tumors.

Quality Assessment of studies of Diagnostic Accuracy Studies (QUADAS), a systematic quality assessment tool designed for diagnostic accuracy testing was applied in the present meta-analysis (Whiting et al., 2006). The QUADAS criteria included 4 domains: patient selection, index test, reference standard, and flow and timing. Each domain is assessed in terms of risk of bias, and the first 3 domains are also assessed in terms of concerns regarding applicability. Where there were discrepancies between the reviewers, a consensus reviewer resolved the differences.

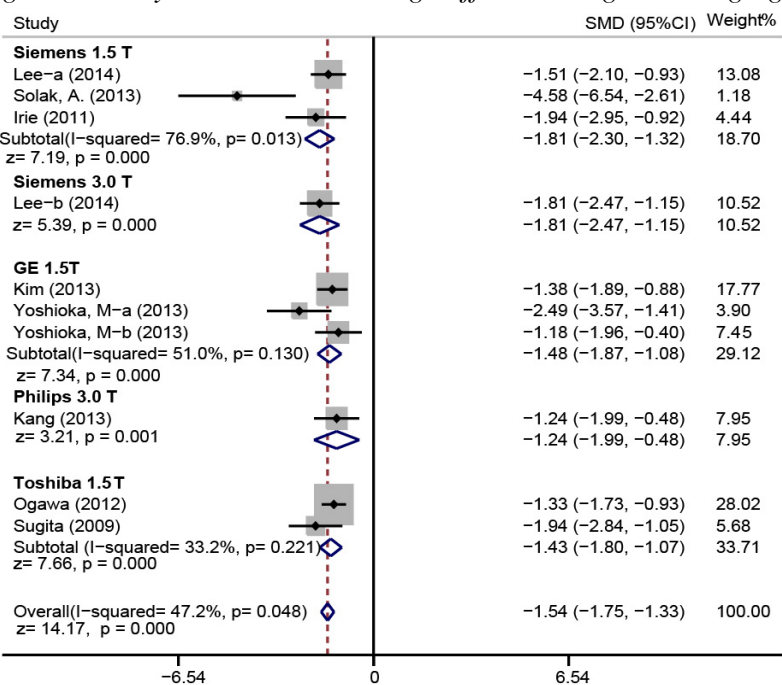

Figure 3. Subgroup Analysis Based on the MRI Machine Type on the Difference of ADC Value Between Benign and Malignant Biliary Tract Tumors

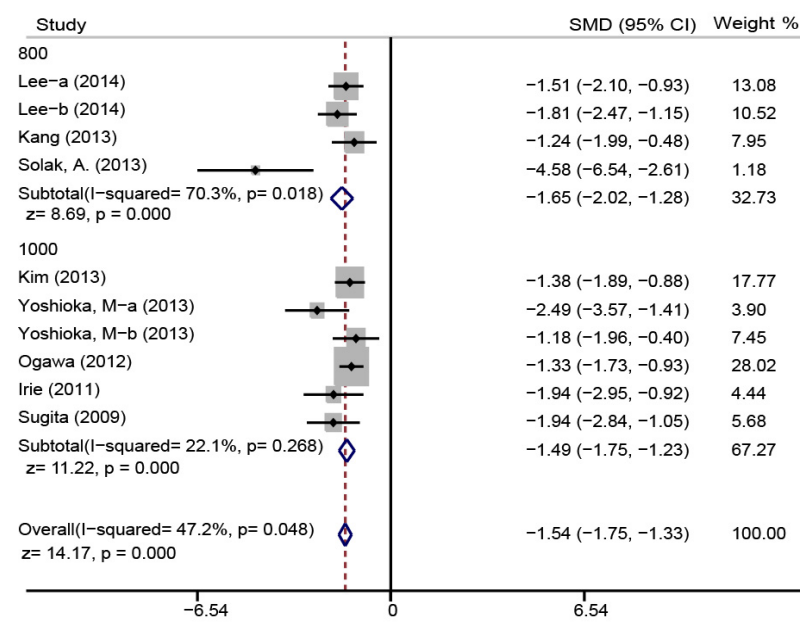

Figure 4. Subgroup Analysis Based on the b Values on the Difference of ADC Value between benign and Malignant Biliary Tract Tumors

\section{Statistical analysis}

Summary standardized mean differences (SMDs) and $95 \%$ confidence intervals $(\mathrm{CI})$ were used to assess the correlation between ADC value of DWI and BTC diagnosis, by utilizing the $\mathrm{Z}$ test. The $\mathrm{Q}$-statistic and I2 test for heterogeneity across the enrolled studies were performed where $P<0.05$ or $\mathrm{I}^{2}>50 \%$ indicated a significant heterogeneity, in which case the random-effects model was employed, or otherwise revealing homogeneity, which required the use of fixed-effects model (Peters et al., 2006; Chen et al., 2012). Publication bias was evaluated with a funnel plot and Egger's linear regression test for each compared model (Sterne and Egger, 2001). All tests were two-sided and a $P$ value of $<0.05$ was regarded as statistically significant. STATA software, version 12.0 (Stata Corp, College Station, TX, USA) was used for statistical analyses.

\section{Results}

Baseline characteristics of included studies

A total of 206 relevant articles were retrieved from 


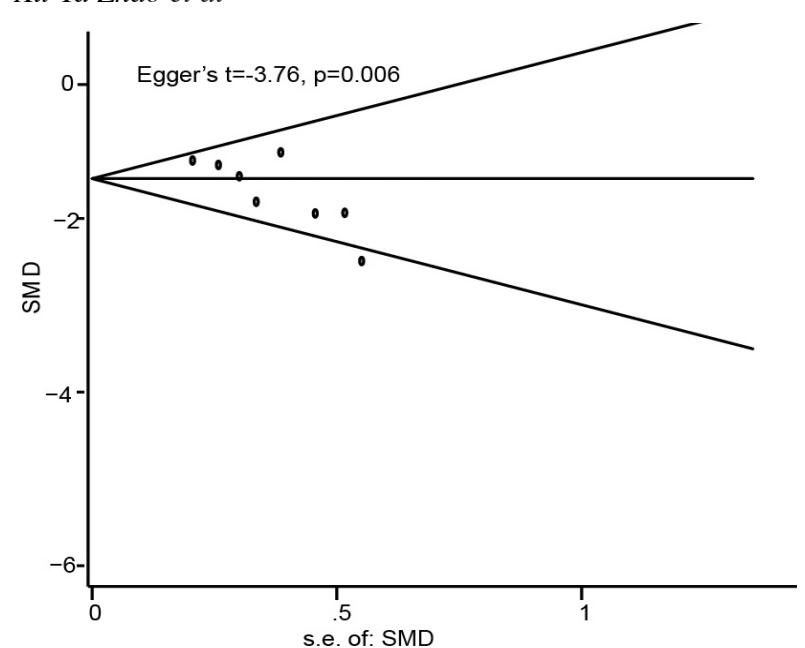

Figure 5. Funnel Plot for Publication Biases on the Difference of ADC Value between Benign and Malignant Biliary Tract Tumors

PubMed ( $\mathrm{n}=35$ ), and EMBASE ( $\mathrm{n}=171)$. After removing the duplicate studies $(n=29)$, the remaining 177 articles were screened by examining titles and abstracts (excluding 102 studies), and following resulting in rejection of 63 articles after full-texts reviewing. Two studies were identified through manual reference search. Leaving 10 studies for further review, two were excluded for insufficient data. Finally, 8 high-quality studies containing 495 benign and malignant tissues were included in our meta-analysis (Sugita et al., 2009a; Irie et al., 2011; Ogawa et al., 2012; Kang et al., 2013; Kim et al., 2013b; Solak et al., 2013; Yoshioka et al., 2013; Lee et al., 2014b). The publication year of the studies ranged between 2009 and 2014. Table 1 and Figure 1 summarize the detailed characteristics and methodological quality of all included studies, respectively.

\section{DWI in the differential diagnosis of BTC}

A total of eight eligible studies reported the use of ADC value of DWI in the diagnosis of BTC. No significant inter-study heterogeneity was detected $\left(\mathrm{I}^{2}=47.2 \%\right.$, $P=0.048)$, therefore fixed-effects model was chosen. As shown in Figure 2, the main results revealed that ADC value in the BTC tissues were significantly lower than that in benign biliary tract tissues (SMD=-1.54, 95\%CI: $-1.75 \sim-1.33, P<0.001)$. Subgroup analysis based on the MRI machine type used showed that the ADC values were consistent, accurate and reliable in the diagnosis of BTC when comparing cancer tissue vs. benign tissue under the instruments of Siemens 1.5 T/3.0 T, Philips 3.0 T, GE 1.5 $\mathrm{T}$, and Toshiba $1.5 \mathrm{~T}$, respectively (all $P<0.05$ ) (Figure 3 ). Further, ADC values were still consistent and accurate in the diagnosis of BTC when comparing cancer tissue vs. benign tissue under the $\mathrm{b}$ value of 800 and $1000 \mathrm{~s} / \mathrm{mm} 2$ (800: $\mathrm{SMD}=-1.65,95 \% \mathrm{CI}:-2.02 \sim 1.28, P<0.001 ; 1000$ : SMD $=-1.49,95 \%$ CI: $-1.75 \sim-1.23, P<0.001$; respectively) (Figure 4).

\section{Publication bias}

For cancer tissue vs. benign tissue comparison models, funnel plot presented to be asymmetry, and Egger's test provided statistical evidence of asymmetry. Therefore, our results suggested a possible evidence of publication bias $(P=0.006$ for cancer tissue vs. benign tissue) (Figure 5).

\section{Discussion}

Application of the ADC values in DWI for the diagnosis and differential diagnosis of human tumors in various organs has been attempted recently. In this meta-analysis, we extracted data from previous studies to investigate the clinical effect of DWI ADC values in differential diagnosis of BTC among Asian populations.

The ADC value is significantly lower in BTC compared to benign biliary tract diseases, suggesting that the $\mathrm{ADC}$ value has a vital significance in discriminating between malignancies and benign diseases, and serve as an important tool for early diagnosis of BTC. The accuracy of DWI detection depends on the calculation of the average ADC values using different $b$ values, reflecting the degree of water protons motion, which relates to tumor size and type, and has been successfully employed to diagnose malignancies of abdominal organs (Somford et al., 2012; Donati et al., 2014; Queiroz et al., 2014). When compared with benign biliary tract diseases, higher signal intensity on DWI and lower ADC values were observed in solid masses, where such values allows for quantitative information gathering that reflect the mobility of tissue water, suggesting that malignant tissues have more resistance to water diffusion, thus decreasing $A D C$ values. $A D C$ values in malignant lesions generally have a decreasing trend, probably due to malignancies usually correlate with the increased tissue cellularity or cell density (Sugita et al., 2009b). Besides the restriction of cellular membranes, intracellular molecules may also result in the restriction of water molecules diffusion in tumors (Le Bihan, 2013). With respect to this, ADC values of DWI are speculated to be associated with the characteristics of different histological tissue types. The variability of DWI is well-known and affects the differential diagnosis of BTC, lowered ADC value is identified in malignant BTC compared with benign biliary tract diseases due to dense cellularity (Tan and Lim, 2013; Park et al., 2014). Additionally, in ampullary carcinoma, a rare carcinoma arising in the ampulla of Vater, the mean ADC value of the ampullary carcinoma group was apparently lower than that of ampullary benignancy group, which indicates the feasibility of DWI and ADC value to functionally evaluate tumors for their aggressiveness response (Jang et al., 2013). Differences in ADC value in BTC is attributed to tumor cell status including higher tumor cellularity, histological tissue types, and extracellular space tortuosity, all of which were linked to different histological characteristics of BTC, consequently, $\mathrm{ADC}$ values have the role in assessing in vivo differential diagnosis of malignancies from benign biliary tract diseases (Padhani et al., 2009; Park et al., 2014). Consistent with our results, Kim et al., found that adding DWI to the standard biliary MRI protocol may improve sensitivity for distinguishing gallbladder cancers from benign gallbladder diseases with wall thickening in Koreas (Kim et al., 2013a). 
Subgroup analysis was performed to take into consideration of other factors such as DWI machine type and different $b$ value that may affect the relation between ADC values and differential diagnosis of BTC. Stratified analysis based on machine type showed that the correlation cannot be influenced by use of Siemens 1.5 T/3.0 T, Philips 3.0 T, GE 1.5 T, and Toshiba 1.5 T. Further, the b value is an index of the degree of motion probing gradient for diffusion weighting, the higher the $b$ value, the more accurate the DWI reflecting the motion of water proton (Malayeri et al., 2011).And our results indicated that ADC values were consistent and accurate in the diagnosis of BTC when comparing cancer tissue vs. benign tissue under the $b$ value of 800 and $1000 \mathrm{~s} / \mathrm{mm} 2$. Our study results are partly consistent with previous studies and show that ADC value of DWI may be helpful for the differential diagnosis of BTC and lower ADC values were associated with the increasing trend of malignant biliary tract lesions.

There were still limitations. First, the numbers of studies were relatively small and the sample size of benign or malignant biliary tract masses were also relatively small, which might have influenced the statistical analysis in determining difference between the ADC values of benign and malignant tumor tissues. Second, retrospective designed meta-analysis might have a negative effect on the optimization of those acquisition parameters like the $b$ value and the $\mathrm{ADC}$ value that contribute to the production of bias affecting the whole results. Third, we concluded a lower ADC value of DWI correlates with the tendency of malignant masses, but the actual cut-off value of ADC for discriminating between malignant and benign lesions, which may restrict the diagnostic accuracy. Fourthly, the potential existence of publication bias might influence the overall estimation. And inter-study heterogeneity where different studies exhibited quite a strong discrepancy may also have a negative influence understanding the results. Finally, severe inflammatory changes related to intramural abscess, congestion, or fibrosis may limit water diffusion in benign biliary tract diseases as well, resulting in overlaps of the ADC values that could lead to a false-positive result in differentiating benign and malignant lesions.

In summary, patients with BTC have low DWI ADC values than in patients with benign biliary tract diseases in Asians. Consequently, ADC values in DWI may be helpful for differentiating BTC from benign biliary tract diseases. Noteworthy, there is a need of larger sample size study for the clarification of this topic in future.

\section{Acknowledgements}

We would like to acknowledge the helpful comments on this paper received from our reviewers.

\section{References}

Akay S, Kocaoglu M, Emer O, et al (2013). Diagnostic accuracy of whole-body diffusion-weighted magnetic resonance imaging with $3.0 \mathrm{~T}$ in detection of primary and metastatic neoplasms. J Med Imaging Radiat Oncol, 57, 274-82.

Attariwala R, Picker W (2013). Whole body MRI: improved lesion detection and characterization with diffusion weighted techniques. J Magn Reson Imaging, 38, 253-68.

Baheti AD, Tirumani SH, Rosenthal MH, et al (2014). Diagnosis and management of intrahepatic cholangiocarcinoma: a comprehensive update for the radiologist. Clin Radiol, 69, 463-70.

Castro FA, Koshiol J, Hsing AW, et al (2013). Biliary tract cancer incidence in the United States-Demographic and temporal variations by anatomic site. Int J Cancer, 133, 1664-71.

Charatcharoenwitthaya $P$, Enders FB, Halling KC, et al (2008). Utility of serum tumor markers, imaging, and biliary cytology for detecting cholangiocarcinoma in primary sclerosing cholangitis. Hepatol, 48, 1106-17.

Chen H, Manning AK, Dupuis J (2012). A method of moments estimator for random effect multivariate meta-analysis. Biometrics, 68, 1278-84.

Chen LD, Xu HX, Xie XY, et al (2008). Enhancement patterns of intrahepatic cholangiocarcinoma: comparison between contrast-enhanced ultrasound and contrast-enhanced CT. Br J Radiol, 81, 881-9.

Donati OF, Chong D, Nanz D, et al (2014). Diffusion-weighted MR imaging of upper abdominal organs: field strength and intervendor variability of apparent diffusion coefficients. Radiol, 270, 454-63.

Furuse J, Kasuga A, Takasu A, et al (2012). Role of chemotherapy in treatments for biliary tract cancer. $J$ Hepatobiliary Pancreat Sci, 19, 337-41.

Gourgiotis S, Kocher HM, Solaini L, et al (2008). Gallbladder cancer. Am J Surg, 196, 252-64.

Heijmen L, Verstappen MC, Ter Voert EE, et al (2012). Tumour response prediction by diffusion-weighted MR imaging: ready for clinical use? Crit Rev Oncol Hematol, 83, 194-207.

Hsing AW, Sakoda LC, Rashid A, et al (2008). Variants in inflammation genes and the risk of biliary tract cancers and stones: a population-based study in China. Cancer Res, $\mathbf{6 8}$, 6442-52.

Irie H, Kamochi N, Nojiri J, et al (2011). High b-value diffusionweighted MRI in differentiation between benign and malignant polypoid gallbladder lesions. Acta radiologica (Stockholm, Sweden : 1987), 52, 236-40.

Jang KM, Kim SH, Lee SJ, et al (2013). Added value of diffusion-weighted MR imaging in the diagnosis of ampullary carcinoma. Radiol, 266, 491-501.

Kang TW, Kim SH, Park HJ, et al (2013). Differentiating xanthogranulomatous cholecystitis from wall-thickening type of gallbladder cancer: Added value of diffusionweighted MRI. Clinical Radiol, 68, 992-1001.

Khoo MM, Tyler PA, Saifuddin A, et al (2011). Diffusionweighted imaging (DWI) in musculoskeletal MRI: a critical review. Skeletal Radiol, 40, 665-81.

Kim JH, Byun JH, Lee SJ, et al (2012). Differential diagnosis of sclerosing cholangitis with autoimmune pancreatitis and periductal infiltrating cancer in the common bile duct at dynamic CT, endoscopic retrograde cholangiography and MR cholangiography. Eur Radiol, 22, 2502-13.

Kim SJ, Lee JM, Kim H, et al (2013a). Role of diffusionweighted magnetic resonance imaging in the diagnosis of gallbladder cancer. J Magn Reson Imag, 38, 127-37.

Kim SJ, Lee JM, Kim H, et al (2013b). Role of diffusionweighted magnetic resonance imaging in the diagnosis of gallbladder cancer. J Magn Reson Imag, 38, 127-37.

Kyriazi S, Collins DJ, Morgan VA, et al (2010). Diffusionweighted imaging of peritoneal disease for noninvasive staging of advanced ovarian cancer. Radiographics, $\mathbf{3 0}$, 1269-85.

Lambrecht M, Van Calster B, Vandecaveye V, et al (2014). Integrating pretreatment diffusion weighted MRI into a 


\section{Xu-Ya Zhao et al}

multivariable prognostic model for head and neck squamous cell carcinoma. Radiother Oncol, 110, 429-34.

Le Bihan D (2013). Apparent diffusion coefficient and beyond: what diffusion MR imaging can tell us about tissue structure. Radiol, 268, 318-22.

Lee NK, Kim S, Kim GH, et al (2012). Diffusion-weighted imaging of biliopancreatic disorders: correlation with conventional magnetic resonance imaging. World $J$ Gastroenterol, 18, 4102-17.

Lee NK, Kim S, Kim TU, et al (2014a). Diffusion-weighted MRI for differentiation of benign from malignant lesions in the gallbladder. Clin Radiol, 69, 78-85.

Lee NK, Kim S, Kim TU, et al (2014b). Diffusion-weighted MRI for differentiation of benign from malignant lesions in the gallbladder. Clin Radiol, 69, 78-85.

Li B, Li Q, Nie W, et al (2014). Diagnostic value of wholebody diffusion-weighted magnetic resonance imaging for detection of primary and metastatic malignancies: a metaanalysis. Eur J Radiol, 83, 338-44.

Low RN, Sebrechts CP, Barone RM, et al (2009). Diffusionweighted MRI of peritoneal tumors: comparison with conventional MRI and surgical and histopathologic findings-a feasibility study. AJR Am J Roentgenol, 193, 461-70.

Malayeri AA, El Khouli RH, Zaheer A, et al (2011). Principles and applications of diffusion-weighted imaging in cancer detection, staging, and treatment follow-up. Radiographics, 31, 1773-91.

Morizane C, Okusaka T, Mizusawa J, et al (2013). Randomized phase II study of gemcitabine plus S-1 versus S-1 in advanced biliary tract cancer: a Japan Clinical Oncology Group trial (JCOG 0805). Cancer Sci, 104, 1211-6.

Ogawa T, Horaguchi J, Fujita N, et al (2012). High b-value diffusion-weighted magnetic resonance imaging for gallbladder lesions: Differentiation between benignity and malignancy. J Gastroenterol, 47, 1352-60.

Onur MR, Ozturk F, Aygun C, et al (2012). Role of the apparent diffusion coefficient in the differential diagnosis of gastric wall thickening. J Magn Reson Imaging, 36, 672-7.

Padhani AR, Liu G, Koh DM, et al (2009). Diffusion-weighted magnetic resonance imaging as a cancer biomarker: consensus and recommendations. Neoplasia, 11, 102-25.

Park HJ, Kim SH, Jang KM, et al (2014). The role of diffusionweighted MR imaging for differentiating benign from malignant bile duct strictures. Eur Radiol, 24, 947-58.

Peters JL, Sutton AJ, Jones DR, et al (2006). Comparison of two methods to detect publication bias in meta-analysis. JAMA, 295, 676-80.

Queiroz MA, Hullner M, Kuhn F, et al (2014). Use of diffusionweighted imaging (DWI) in PET/MRI for head and neck cancer evaluation. Eur J Nucl Med Mol Imaging, 41, 2212-21.

Randi G, Malvezzi M, Levi F, et al (2009). Epidemiology of biliary tract cancers: an update. Ann Oncol, 20, 146-59.

Shin HR, Oh JK, Masuyer E, et al (2010). Comparison of incidence of intrahepatic and extrahepatic cholangiocarcinoma--focus on East and South-Eastern Asia. Asian Pac J Cancer Prev, 11, 1159-66.

Solak A, Solak I, Genc B, et al (2013). The role of diffusionweighted examination in non-polyploid gallbladder malignancies: A preliminary study. Turkish J Gastroenterol, 24, 148-53.

Somford DM, Hambrock T, Hulsbergen-van de Kaa CA, et al (2012). Initial experience with identifying high-grade prostate cancer using diffusion-weighted MR imaging (DWI) in patients with a Gleason score $</=3+3=6$ upon schematic TRUS-guided biopsy: a radical prostatectomy correlated series. Invest Radiol, 47, 153-8.
Sterne JA, Egger M (2001). Funnel plots for detecting bias in meta-analysis: guidelines on choice of axis. J Clin Epidemiol, 54, 1046-55.

Sugita R, Yamazaki T, Furuta A, et al (2009a). High b-value diffusion-weighted MRI for detecting gallbladder carcinoma: Preliminary study and results. Eur Radiol, 19, 1794-8.

Sugita R, Yamazaki T, Furuta A, et al (2009b). High b-value diffusion-weighted MRI for detecting gallbladder carcinoma: preliminary study and results. Eur Radiol, 19, 1794-8.

Tan CH, Lim KS (2013). MRI of gallbladder cancer. Diagn Interv Radiol, 19, 312-9.

Vilana R, Forner A, Bianchi L, et al (2010). Intrahepatic peripheral cholangiocarcinoma in cirrhosis patients may display a vascular pattern similar to hepatocellular carcinoma on contrast-enhanced ultrasound. Hepatol, 51, 2020-9.

Wen H, Yoo SS, Kang J, et al (2010). A new NMR-based metabolomics approach for the diagnosis of biliary tract cancer. J Hepatol, 52, 228-33.

Whiting PF, Weswood ME, Rutjes AW, et al (2006). Evaluation of QUADAS, a tool for the quality assessment of diagnostic accuracy studies. BMC Med Res Methodol, 6, 9.

$\mathrm{Wu} \mathrm{LM}, \mathrm{Xu} \mathrm{JR}, \mathrm{Lu}$ Q, et al (2013). A pooled analysis of diffusion-weighted imaging in the diagnosis of hepatocellular carcinoma in chronic liver diseases. J Gastroenterol Hepatol, 28, 227-34.

Yoshioka M, Watanabe G, Uchinami H, et al (2013). Diffusionweighted MRI for differential diagnosis in gallbladder lesions with special reference to ADC cut-off values. HepatoGastroenterol, 60, 692-8. 